\title{
Toward time resolved 4D cardiac CT imaging with patient dose reduction: estimating the global heart motion
}

\author{
Katsuyuki Taguchi ${ }^{1}$, W. Paul Segars ${ }^{1}$, George S.K. Fung ${ }^{1,2}$, and Benjamin M.W. Tsui ${ }^{1}$ \\ ${ }^{1}$ The Russell H. Morgan Department of Radiology and Radiological Science, \\ Johns Hopkins University, Baltimore, MD \\ ${ }^{2}$ Department of Electrical and Electronic Engineering, \\ University of Hong Kong, Pokfulam Rd, Hong Kong \\ (Phone: 410-502-3934; Email:ktaguchi@jhmi.edu)
}

\begin{abstract}
Coronary artery imaging with multi-slice helical computed tomography is a promising noninvasive imaging technique. The current major issues include the insufficient temporal resolution and large patient dose. We propose an image reconstruction method which provides a solution to both of the problems. The method uses an iterative approach repeating the following four steps until the difference between the two projection data sets falls below a certain criteria in step-4: 1) estimating or updating the cardiac motion vectors, 2) reconstructing the time-resolved 4D dynamic volume images using the motion vectors, 3) calculating the projection data from the current 4D images, 4) comparing them with the measured ones. In this study, we obtain the first estimate of the motion vector. We use the 4D NCAT phantom, a realistic computer model for the human anatomy and cardiac motions, to generate the dynamic fan-beam projection data sets as well to provide a known truth for the motion. Then, the halfscan reconstruction with the sliding time-window technique is used to generate cine images: $f(t, \underline{r})$. Here, we use one heart beat for each position $\underline{r}$ so that the time information is retained. Next, the magnitude of the first derivative of $f(t, \underline{r})$ with respect to time, i.e., $|d f / d t|$, is calculated and summed over a region-of-interest (ROI), which is called the mean-absolute difference (MAD). The initial estimation of the vector field are obtained using MAD for each ROI. Results of the preliminary study are presented.
\end{abstract}

Keywords: computed tomography $(\mathrm{CT})$, cardiac CT, temporal resolution, radiation dose

\section{INTRODUCTION}

Coronary artery imaging with multi-slice helical computed tomography (CT) is a promising noninvasive imaging technique (1-4). In the early stages of the development of this technique the application of multi-slice CT to cardiac imaging was proposed by Taguchi and Anno (5), Ohnesorge, et al. (6), and others (7)-(8). The quality of the image has been improved with the increases in the gantry rotation speed, the number of detector rows, and the detector height (or $z$ coverage) with the best $z$-resolution: they are currently 330-400 msec/rotation, 32-64 rows for 20-40 mm coverage with 0.4-0.6 mm resolution, respectively (9-11). This provides a good temporal resolution $(50-150 \mathrm{~ms})$ and a good spatial resolution $\left[\sim(0.5 \mathrm{~mm})^{3}\right]$ if the object stays still. The scan duration is as short as 5-10 seconds for the whole heart and the contrast resolution of a reconstructed image is sufficient to depict vulnerable soft plaques in the coronaries.

The usefulness of CT imaging has been demonstrated by clinical studies, especially to rule out the coronary artery diseases and to eliminate unnecessary diagnostic catheter coronary angiography procedures. Raff et al. obtained excellent specificity, sensitivity, and positive and negative predictive values of $95 \%, 90 \%, 93 \%$, and $93 \%$, respectively, for the presence of significant stenosis on a per-patient basis (12). The results of studies have been improving as the performance of the scanners has gotten better. Other potential applications have been investigated which include calcium scoring and the evaluation of cardiac function, myocardial perfusion, infarcts, tumors, pericardial disease, post-surgical complications and congenital malformations $(13,14)$. Some applications use different criteria for patient selection and require heart rates higher than 90 or 100 beats-per-minute (bpm); thus, it is important to improve the temporal resolution.

The current problems with cardiac CT images include the large radiation dose to the patient $(13,16-18)$, residual motion artifacts (1), region dependent optimal cardiac phase (which does not exist in many cases), "banding artifacts" observed in multiplanar reformatted (MPR) or three-dimensional (3D) images $(1,3,19,20)$, and blooming or shading artifacts near large calcium deposits. Among them, the first three are the biggest issues in the current and the future applications. In this study we propose an image reconstruction method which provides a solution to those problems.

Medical Imaging 2006: Physics of Medical Imaging, edited by Michael J. Flynn, Jiang Hsieh, Proceedings of SPIE Vol. 6142, 61420J, (2006) $\cdot 1605-7422 / 06 / \$ 15 \cdot$ doi: 10.1117/12.653279 
The structure of the paper is as follows. In Sec. 2, we review the current cardiac CT imaging and discuss two most important problems, the patient radiation dose and the temporal resolution. In Sec. 3, we propose a new class of iterative, analytical cardiac reconstruction algorithm to solve the problem. We also outline the method to obtain the initial estimation of the motion vector field. Preliminary evaluation is presented in Sec. 4 and Sec. 5 summarizes this paper.

\section{CARDIAC IMAGING}

\subsection{Current strategy}

In most cases, a helical scan is employed with very slow helical pitches, typically $0.2-0.3$ (/rev) while the electrocardiogram (ECG) signal is recorded in parallel. Usually, it takes 5-10 seconds to cover the entire heart. Then, a retrospectively ECG-gated cone-beam helical reconstruction is employed to obtain the volume-of-interest at the cardiac phase of interest. Let us review the current ECG-gated reconstruction algorithm in general. First, cardiac time windows are defined for all of the heart beats (cycles), centering the same phase of the ECG data. Then, helical projection data within the time windows are extracted and used in the image reconstruction.

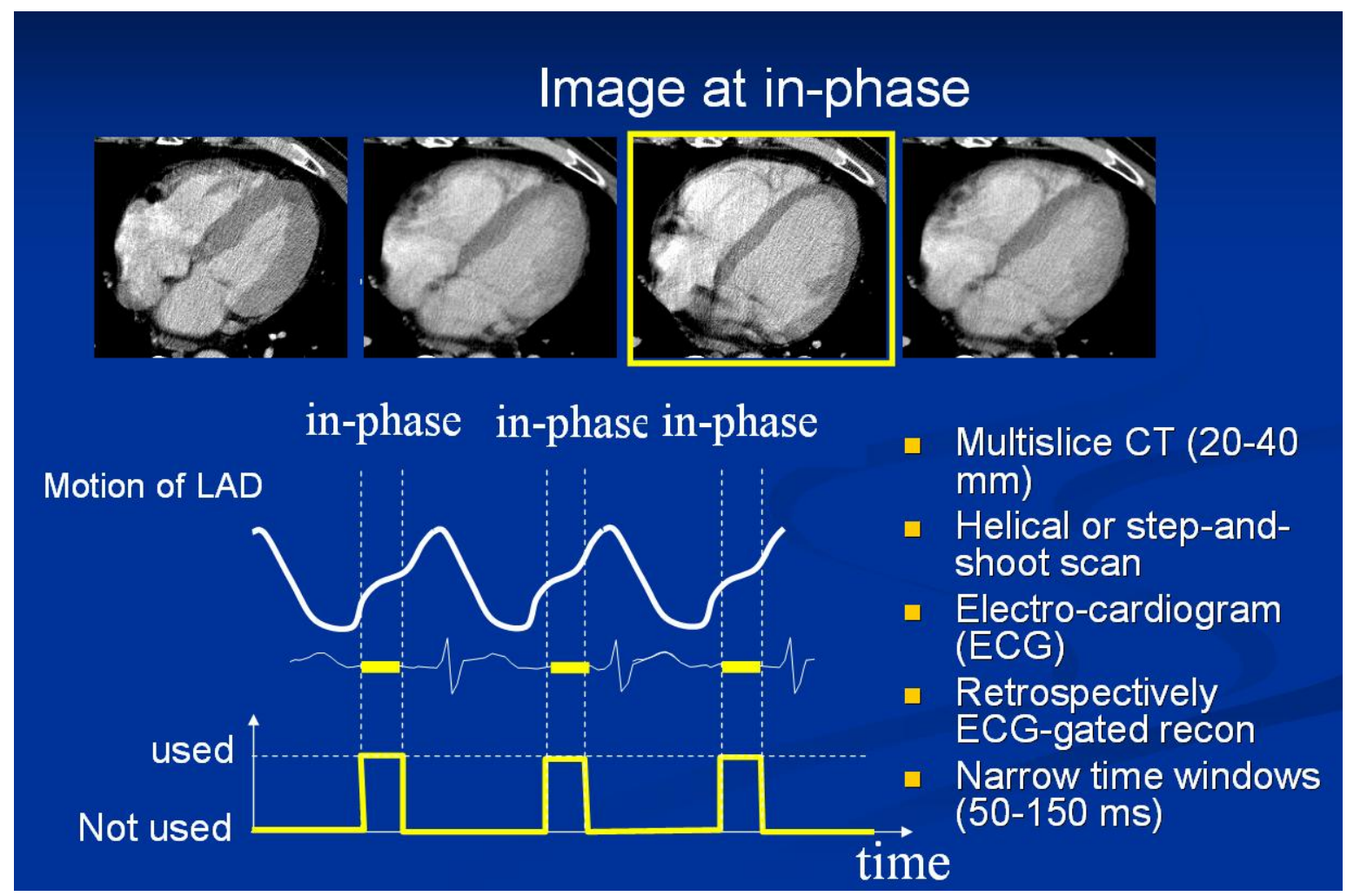

Figure 1. The strategy of the urrent cardiac x-ray CT

There are some variations in methods proposed and implemented in medical x-ray CT scanners $(5,6,8,19,21-24)$. For example, the number of heart beats that contributes to a voxel ranges from 1 to 4 . However, all of the algorithms share a common drawback. They assume the heart does not move within the cardiac time window (50-200 ms), which is not true even though the cardiac phase of interest with slow heart motion (e.g., mid-diastolic phase or the end-systole) is carefully chosen. Or, in other words, the time window width has to be narrow enough so that the assumption is practically acceptable.

The temporal resolution is primarily limited by the rotation time of the gantry. It is further improved by applying narrower time windows and more than one heart beat per voxel. However, applying narrower time windows forces us to throw away more off-phase data (outside of windows), which leads to more patient radiation dose. 


\subsection{Patient radiation dose}

The patient's radiation dose ranges 10-15 mSv in the current cardiac CT examination. Among them, only about 20-30\% of the data are used to reconstruct one cardiac phase of interest. Therefore, the patient radiation dose for cardiac imaging is about 5 times as large as other applications. If we can enlarge the time window width by using the proposing method and increase the data usage ratio, we can reduce the image noise. Then, if the accuracy of the motion estimation is not significantly affected by the amount of image noise, we will be able to reduce the dose while maintaining the image noise at the same level.

A prospective ECG-gated helical scan, which reduces the tube current for off-phase, has been introduced a while ago. It works fine with phantom studies; however, there seems to be practical and mental hurdles for implementing the scheme to the clinical routine. The reasons include that 1 ) the best cardiac phase has to be known before the scan; 2) the heart rate must be very stable; and 3) there are not many cases that satisfy the two conditions. So, it may not be fair to assume the mA modulation be the "current" level; but let us carry out the estimation for both cases.

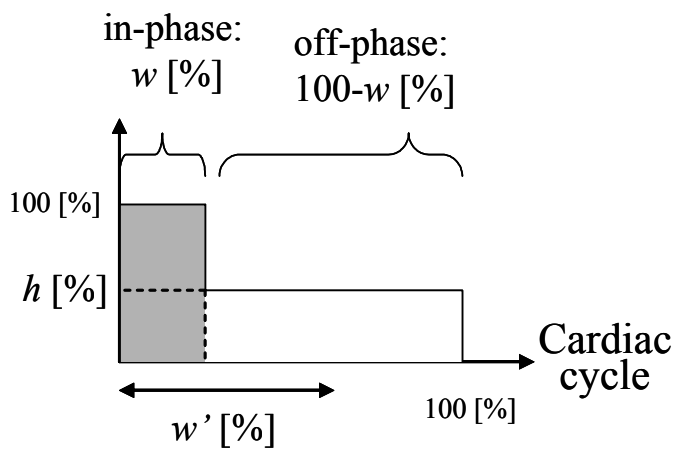

Figure 2. Parameters for the prospective ECG-gated tube current modulation. $w$ refers to a ratio of inphase window to one heart cycle, which has full-power exposure, $h$ denotes the ratio of tube current reduced for the off-phase window, respectively. $w$ ' refers to the expected enlarged time window by the proposed algorithm.

We describe the tube current modulation using two parameters: $w$ and $h . w$ refers to the width of in-phase with full tube current while $h$ is the reduced tube current for off-phase (Figure 2). $w$ was fixed at $25 \%$ as the current standard. We used three $h$ values for $\mathrm{mA}$ modulations: $100 \%, 50 \%$ and 20\%. $h=100 \%$ means no mA modulation is employed; $h=50 \%$ and $20 \%$ are a conservative and an aggressive modulation, respectively. The parameter $w$ ' refers to the time window width enlarged by the proposed method. Here, we used $w^{\prime}=100 \%$ and $50 \%$ for the "ambitious" and "pessimistic" achievement level, respectively.

The image noise can be described by the area of the cardiac time window, $1 / \sqrt{w}$ and $1 / \sqrt{w+h\left(w^{\prime}-w\right)}$ for the current and the proposed method, respectively. The expected noise level (in terms of the standard deviation) with the same radiation dose is shown from the second right column in Table I. The noise SD can be reduced to $50 \%$ of the current level (without modulation) if all of the data can be used for reconstruction. Even with the pessimistic achievement level, it can be $71 \%$ of the current level. They will be a drastic change of the image quality. The advantage is reduced if we combine it with the mA modulation; the noise still can be reduced to $63-89 \%$, which will be also a significant achievement.

Table I. Expected level of the image noise (standard deviation) and radiation dose while the other is fixed at the current level. Values are the percentage of the current level. $w$ is fixed at $25 \%$.

\begin{tabular}{|c|c|c|c|}
\hline$h:$ mA for off-phase & $w^{\prime}:$ Time widow width & Noise SD & Dose \\
\hline $100 \%$ (no modulation) & $100 \%$ & $50 \%$ & $25 \%(3 \mathrm{mGy})$ \\
\hline $100 \%$ (no modulation) & $50 \%$ & $71 \%$ & $50 \%(6 \mathrm{mGy})$ \\
\hline $50 \%$ & $100 \%$ & $63 \%$ & $40 \%$ \\
\hline $50 \%$ & $50 \%$ & $82 \%$ & $67 \%$ \\
\hline $20 \%$ & $100 \%$ & $76 \%$ & $57 \%$ \\
\hline $20 \%$ & $50 \%$ & $89 \%$ & $80 \%$ \\
\hline
\end{tabular}


Estimation of the dose reduction is not straightforward because it seems reasonable to assume that the accuracy of the motion estimation is affected by the level of the noise in cine images. Acknowledging the non-linearity issue, let us go ahead and make estimation. The goal is to maintain the image noise, thus, to keep the area of the cardiac time window with the enlarged window width $w^{\prime}$. Therefore, the required dose ratio can be calculated by $w /\left[w+h\left(w^{\prime}-w\right)\right]$. Results are shown in the right-most column in Table I. The radiation dose could be as low as $25 \%$ of the current level (no tube current modulation). Note that, even though we reduce the radiation dose, this approach will not sacrifice either of the spatial resolution, image noise, or temporal resolution.

\subsection{Temporal resolution}

The spatial resolution of the reconstructed clinical images is an attribution of the "ideal spatial resolution" and the temporal resolution: It may be the result of a convolution of two profiles, where the ideal spatial resolution refers to the three-dimensional spatial resolution obtained when the object is still. Therefore, when the object moves as the case of cardiac imaging, a method with better temporal resolution frequently gives better image quality. Thus, improving the temporal resolution would improve the sharpness of the images of the heart.

The heart keeps moving even in the most "quiet" phases (e.g., mid-diastole); thus, employing a reconstruction algorithm without motion compensation always results in blurred spatial resolutions due to the (motion induced) inconsistency even within the narrow time windows. Thus, we propose a new class of algorithm which estimates the motion vectors of the object and utilizes them in the deformation reconstruction technique. With the proposed method, the temporal resolution of the cardiac images is defined by the overall accuracy of the estimated motion vector field within the cardiac time window; it is not a function of the window width itself. Well, how far could the temporal resolution improve? Let us leave the answer to the future investigation.

\section{TIME RESOLVED 4D CARDIAC RECONSTRUCITON ALGORITHM}

\subsection{General framework}

The proposed algorithm uses an iterative approach which repeats the following four steps until the difference between the two projection data sets falls below a certain criteria in step-4 (Figure 3): 1) estimating or updating the cardiac motion vectors, 2) reconstructing the time-resolved 4D dynamic volume images using the motion vectors, 3) calculating the projection data from the current $4 \mathrm{D}$ images, 4) comparing them with the measured projection data sets. The temporal resolution will be limited by the accuracy of the estimated motion vector fields within the time window.

Cone-beam projections measured along a helical orbit are given by $g^{m}(t, \underline{\Theta})=\int_{-\infty}^{\infty} f(t, \underline{s}(\lambda)+l \underline{\Theta}) d l$, where $f(t, \underline{r})$ is the dynamic object to reconstruct, $\underline{\Theta}$ denote a unit vector directed from the x-ray focus $\underline{s}(\lambda)$ where $\lambda$ is a source-parameter which is related to time $t$.

In the first step, the initial estimate of the motion vector $v^{(0)}(t, \underline{r})$ is obtained using the method describe later, and then, the previous estimate $v^{(k-1)}(t, \underline{r})$ is updated in the $k^{\text {th }}$ iteration in order to minimize the difference between the calculated and the measured projection data sets. This study focuses on the initial motion estimation.

In the second step, the analytical reconstruction with the time dependent deformation technique (25-28) will be adapted to the ECG-gated cone-beam helical reconstruction algorithm (19) in order to incorporate the motion of the object. Using the measured projection data and the current estimate of the motion vector $v^{(k)}(t, \underline{r})$, we obtain the dynamic volume data, $f^{(k)}(t, \underline{r})$. The third step can be done numerically to obtain the time resolved cone-beam projection data, $g^{(k)}(t, \underline{\Theta})=\int_{-\infty}^{\infty} f^{(k)}(t, \underline{s}(\lambda)+l \underline{\Theta}) d l$. In the fourth step, the obtained data are compared with the measured data, $g^{m}(t, \underline{\Theta})$. Note that only a subset of $\mathrm{g}^{(k)}$ has their counterparts since $\lambda$ in $\mathrm{g}^{m}$ is $t$-dependent. When the iteration converges, $g^{(k)}(t, \underline{\Theta}) \approx g^{m}(t, \underline{\Theta})$, we obtain a good estimation of motion vectors to use out-of-phase data $\left.g^{m}\left(t^{\prime}, \underline{\Theta}\right)\right|_{t^{\prime} \neq t}$ to reconstruct the object at the phase-of-interest $f(t, \underline{r})$. This allows us to enlarge the time windows for reducing the image noise (thus, reducing the patient dose keeping the image noise at the same level) while improving the time resolution. 


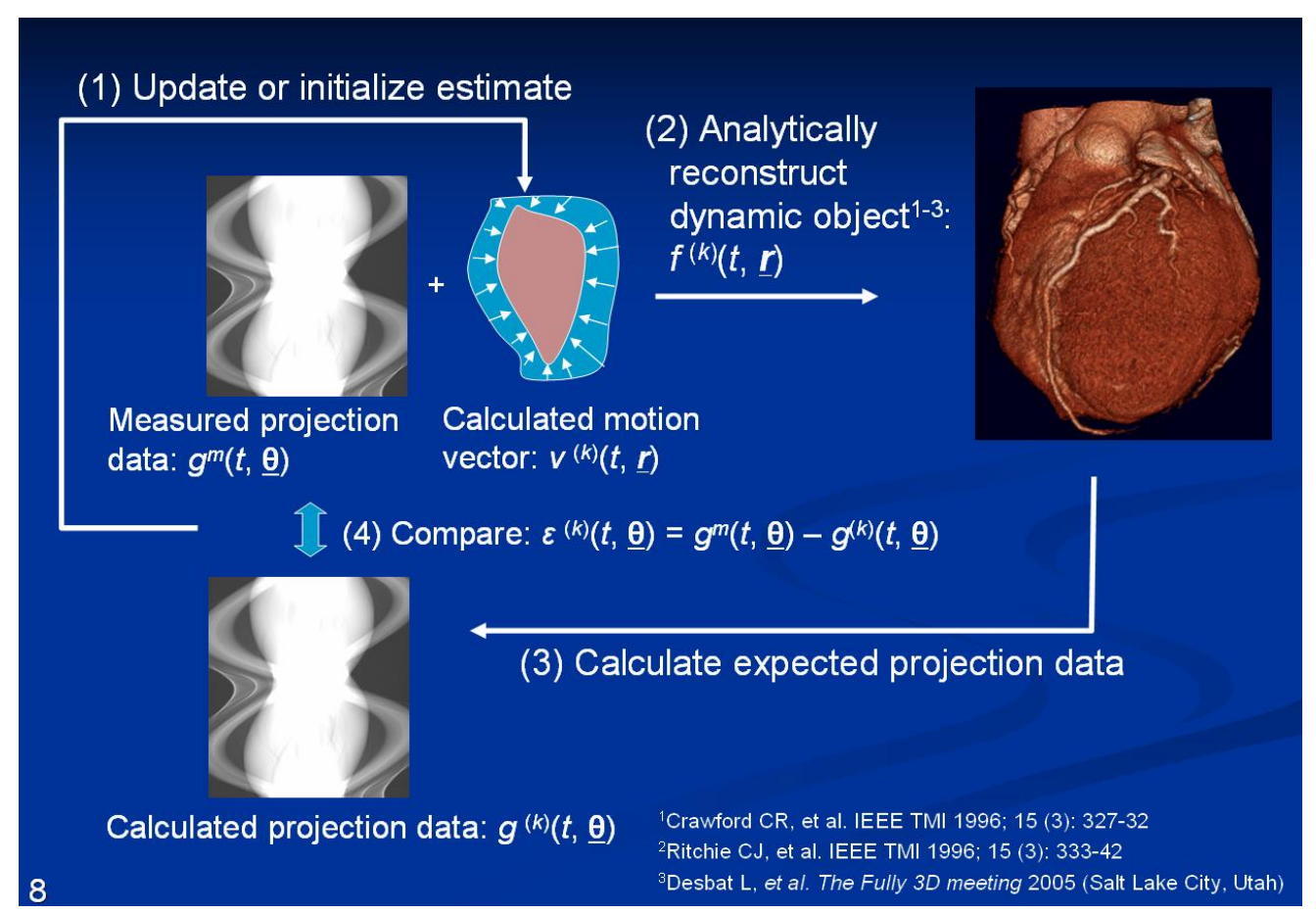

Figure 3. The proposed algorithm (general framework)

\subsection{Initial estimation of the motion vector field}

In this study, we obtain the initial estimate of the motion vector. Four-dimensional cine images $f^{(0)}(t, \underline{r})$ are reconstructed by sliding a time window along the time-axis (5) (Figure 4, left). We use Parker weight (29) for the time window function. Here, we use one heart beat for each position $\underline{r}$ so that the time information is retained (5). The motion vector field is estimated in the next step; there are two candidate approaches: A sequential method and the sandwich method. The sequential method use subsequent two cine frames sequentially. In contrast, the sandwich method uses two quiet phases, the end-systole and the mid-diastole, as two end-points to roughly estimate the motion vectors and gradually increase the accuracy of the estimation by using middle frames.

In either method, we use the template matching technique with the mean-absolute difference (MAD) to obtain the motion vector field. CT images have a lot of landmarks that could help the estimation. We deal with two frames at a time. First, ROI's (macro-blocks) are uniformly distributed $(50 \times 50)$ in the current frame. Next, in the reference frame, we limit the search area and move around the ROI within the area. The mean-absolute difference is calculated at each location. The least MAD value indicates the best match between two frames; that defines the motion vector of the ROI. We repeat the same process for all of the ROIs.

MAD is the average magnitude of the first derivative (or gradient) defined by $\int_{S}\left|d f^{(0)}(t, \underline{r}) / d t\right| d \underline{r}$, where $S$ is the volumeof-interest. The small region $S$ is used for template matching (above) while $S$ is the entire image for the sandwich approach (30) to obtain the global heart motion index, thus, to define two end-points for initial estimate of the motion vectors $\underline{v}^{(0)}(t, \underline{r})$. 


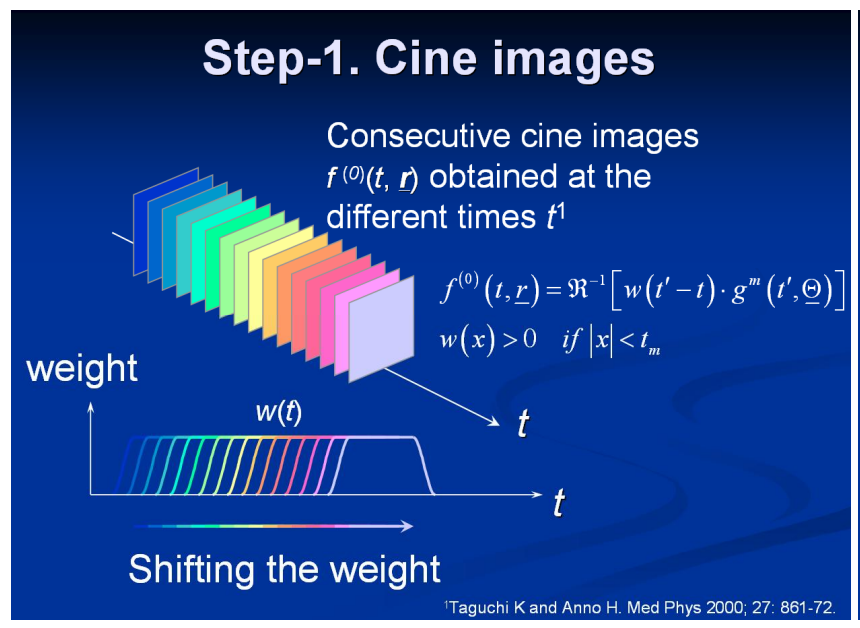

\section{Sequential vs Sandwich methods}

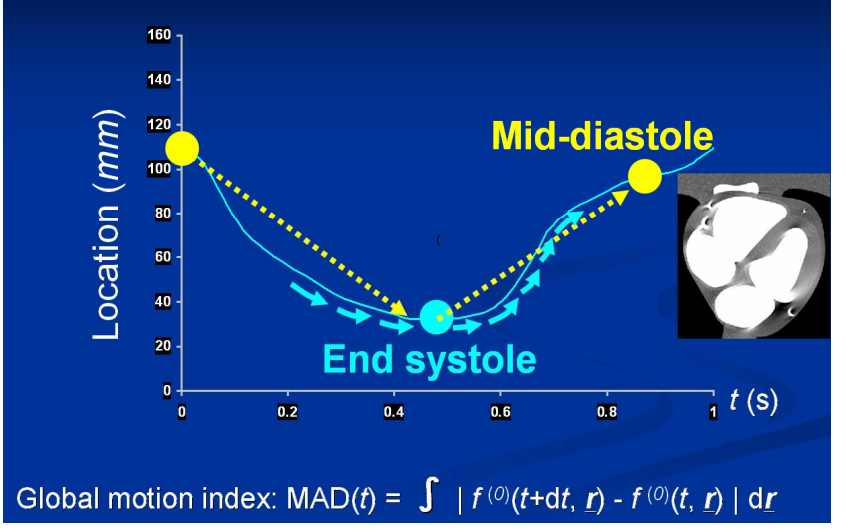

Figure 4. Initial motion estimation. First, cine images are reconstructed (left). Then, there are two approaches for the initial estimate of the motion vector field: sequential or sandwich approaches (right).

\section{EVALUATIONS}

We used the 4D Non-uniform rational B-spline based Cardiac-Torso (NCAT) phantom (31-33) version 2.0 to generate the dynamic fan-beam projection data sets as well as the known truth for the motion patterns. The heart rate was 60 bpm and the body size was "normal." The middle of the heart, where the motion is quasi-two dimensional, was scanned by $300 \mathrm{~ms} / \mathrm{rev}$ for 10 rotations. The scanner was a single-slice fan-beam CT with a typical geometry of medical scanners rotating. No noise was added.

Cine images with a 512 × 512 for $200 \times 200 \mathrm{~mm}$ were reconstructed by Parker weight with a time increment of $20 \mathrm{~ms}$ over two heart beats. The method described above is applied to obtain the initial estimation of the motion vector field. Note that for this preliminary study, we assumed the motion was in two dimensions ( $x$ and $y$ ), or in other words, we are estimating the $x y$ components of the motion vector.

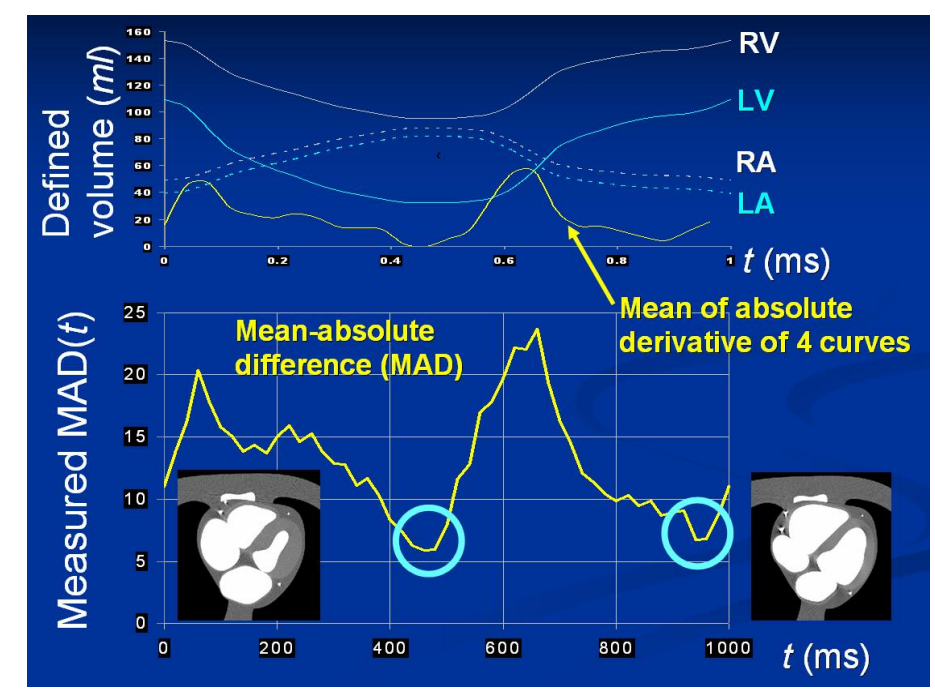

Figure 5. The mean-absolute difference (MAD) provides two quiet phases, endsystole (ES) and mid-diastole (MD), which can be two end points of global motion vectors. 
Figure 5 on the top are the time-volume curves of four chamber of the NCAT phantom calculated from the definitions. The curve of the left ventricle shows the end-systole and the mid-diastole at $45 \%$ and $81 \%$, respectively. We calculated the derivative of the each curve and simply averaged them to obtain the yellow curve: The bottom graph is the MAD obtained from cine images. You can clearly see the similarity between two curves. There are fast motion phases at the beginning of the contraction $(t \approx 50 \mathrm{~ms})$ and during expansion $(t \approx 650 \mathrm{~ms})$ while two quiet points can be recognized exactly at the end-systole $(t \approx 450 \mathrm{~ms})$ and near the mid-diastole $(t \approx 960 \mathrm{~ms})$. The reason of the minor disagreement at the mid-diastole is still under investigation. Anyway, these results supported the idea of using MAD to find two quiet phases in general.

Figure 6 shows the estimated motion vector field by the sandwich method using two frames at the end-systole and the mid-diastole. The motion of the myocardium or the heart wall were correct; the left anterior descending artery was tracked; and so as the left circumflex. The motion of the right coronary artery (RCA) (Figure 5, yellow boxes) from the mid-diastole to the end-systole (right) was also correctly estimated, however, from the systole to the diastole, the vectors were pointing to wrong directions. It happens when there is no constraint applied both in space and time.

The sequential approach showed a similar tendency. The wall motion was accurately estimated while motion of the RCA was not accurate. It was because the motion artifact was too severe.

This paper demonstrated the usefulness of the 4D NCAT phantom in the development of the dynamic volumetric imaging since we know the true shape of the object and its motion vector at every voxel and time point. The realistic shape and the contrast of the 4D NCAT phantom will allow us to evaluate the significance of the artifact, which was not feasible with other phantoms.

\section{SUMMARY}

We proposed a novel iterative dynamic reconstruction algorithm for coronary artery imaging with multi-slice helical computed tomography. The method estimates the motion vector fields and uses it during the dynamic reconstruction. The result of the pilot study for the first step was not perfect, but seemed to have good agreement with the truth. If the proposed method is completed, it will resolve most of the current issues in cardiac x-ray CT such as large patient radiation dose. It will also provide much sharper images and open the possibility for new applications. We will further develop the proposed algorithm.

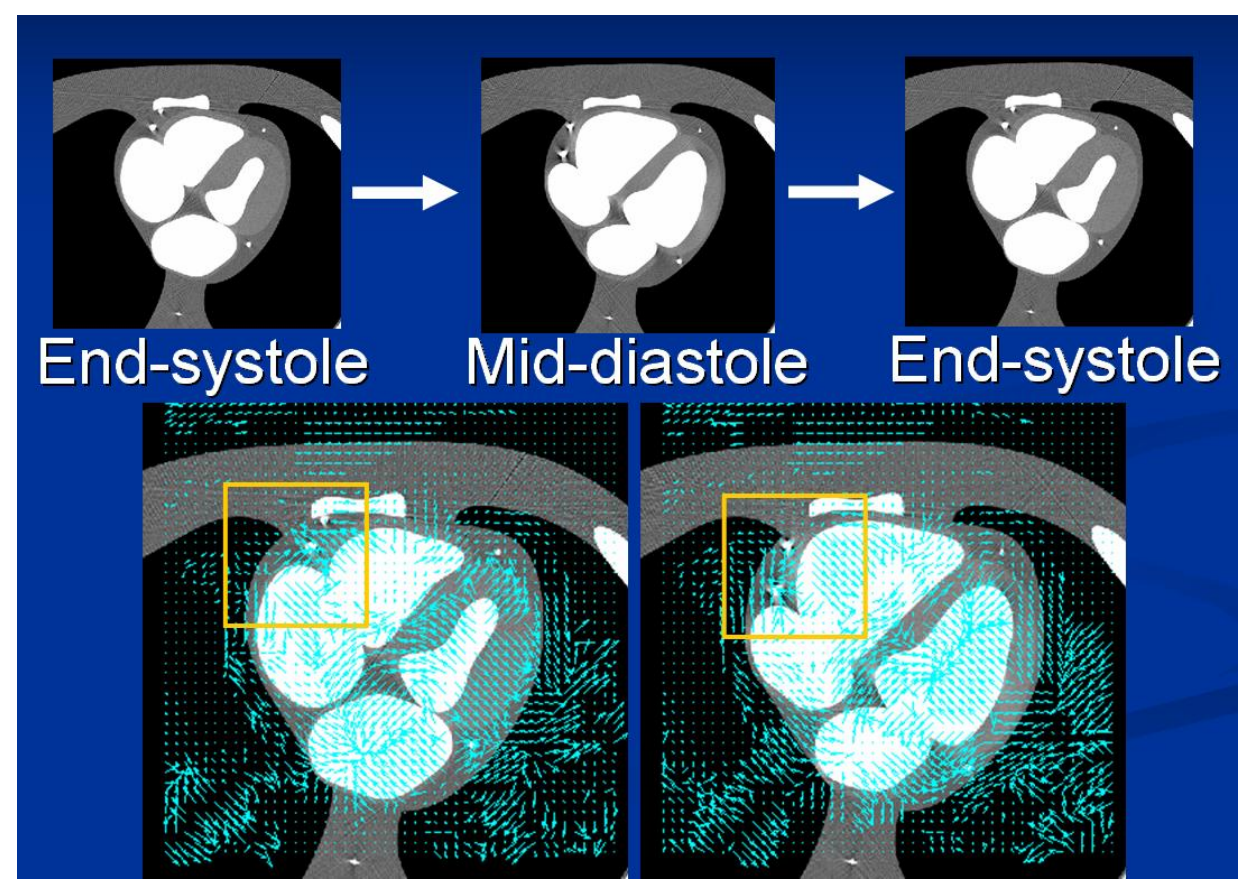

Figure 6. Estimated motion vector field from two frames (ES and MD). 


\section{ACKNOWLEDGMENT}

We thank Drs. Elliot K. Fishman, Hirofumi Anno and Ilmar A. Hein for their indirect contribution to this study. The work is supported in part by NIH grant R01 EB001838 and the start-up fund of the Division of Medical Imaging Physics at the Russell H. Morgan Department of Radiology and Radiological Science in the Johns Hopkins University.

\section{REFERENCES}

1. Hoffmann MH, Shi H, Manzke R, Schmid FT, De Vries L, Grass M, et al. Noninvasive coronary angiography with 16-detector row CT: effect of heart rate. Radiology. 2005 Jan;234(1):86-97.

2. Burgstahler C, Beck T, Kuettner A, Reimann A, Kopp AF, Heuschmid M, et al. Image quality and diagnostic accuracy of 16-slice multidetector spiral computed tomography for the detection of coronary artery disease in elderly patients. J Comput Assist Tomogr. 2005 Nov-Dec;29(6):734-8.

3. Hofmann LK, Zou KH, Costello P, Schoepf UJ. Electrocardiographically gated 16-section CT of the thorax: cardiac motion suppression. Radiology. 2004 Dec;233(3):927-33.

4. Chen G-H, Tokalkanahalli R, Zhuang T, Nett BE, Hsieh J. Development and evaluation of an exact fan-beam reconstruction algorithm using an equal weighting scheme via locally compensated filtered backprojection (LCFBP). Medical Physics. 2006;33(2):475-81.

5. Taguchi K, Anno H. High temporal resolution for multislice helical computed tomography. Med Phys. 2000 May;27(5):861-72.

6. Ohnesorge B, Flohr T, Becker C, Kopp AF, Schoepf UJ, Baum U, et al. Cardiac imaging by means of electrocardiographically gated multisection spiral CT: initial experience. Radiology. 2000 Nov;217(2):564-71.

7. Hui H, Tinsu P, Yun S. Multislice helical CT: image temporal resolution. Medical Imaging, IEEE Transactions on. 2000;19(5):384-90.

8. Kachelriess M, Ulzheimer S, Kalender WA. ECG-correlated image reconstruction from subsecond multi-slice spiral CT scans of the heart. Med Phys. 2000 Aug;27(8):1881-902.

9. Hoffmann MH, Lessick J, Manzke R, Schmid FT, Gershin E, Boll DT, et al. Automatic determination of minimal cardiac motion phases for computed tomography imaging: initial experience. Eur Radiol. 2005 Jul 14.

10. Flohr TG, Schaller S, Stierstorfer K, Bruder H, Ohnesorge BM, Schoepf UJ. Multi-detector row CT systems and image-reconstruction techniques. Radiology. 2005 Jun;235(3):756-73.

11. Sablayrolles JL, Cesmeli E, Mitandjian L, Adda O, Dessalles-Martin D. Wide coverage by volume CT: Benefits for cardiac imaging. Proceedings of SPIE: Medical Imaging 2005: Physics of Medical Imaging. 2005;5745:324-31.

12. Raff GL, Gallagher MJ, O'Neill WW, Goldstein JA. Diagnostic accuracy of noninvasive coronary angiography using 64-slice spiral computed tomography. J Am Coll Cardiol. 2005 Aug 2;46(3):552-7.

13. Desjardins B, Kazerooni EA. ECG-gated cardiac CT. AJR Am J Roentgenol. 2004 Apr;182(4):993-1010.

14. Gilkeson RC, Ciancibello L, Zahka K. Pictorial essay. Multidetector CT evaluation of congenital heart disease in pediatric and adult patients. AJR Am J Roentgenol. 2003 Apr;180(4):973-80.

15. Flohr TG, Schoepf UJ, Kuettner A, Halliburton S, Bruder H, Suess C, et al. Advances in cardiac imaging with 16section CT systems. Acad Radiol. 2003 Apr;10(4):386-401.

16. Flohr TG, McCollough CH, Bruder H, Petersilka M, Gruber K, Subeta C, et al. First performance evaluation of a dual-source CT (DSCT) system. Eur Radiol. 2005 Dec 10.

17. Morin RL, Gerber TC, McCollough CH. Radiation dose in computed tomography of the heart. Circulation. 2003 Feb 18;107(6):917-22.

18. Vrtiska TJ, Fletcher JG, McCollough CH. State-of-the-art imaging with 64-channel multidetector CT angiography. Perspect Vasc Surg Endovasc Ther. 2005 Mar;17(1):3-8; discussion 9-10.

19. Taguchi K, Chiang BS, Hein IA. Direct cone-beam cardiac reconstruction algorithm with cardiac banding artifact correction. Medical Physics. 2006;33(2):521-39.

20. Shechter G, Levi I, Altman A. Robust band artifact suppression for cardiac CT. IEEE Medical Imag Conf. 2005 Oct:M10-4.

21. Flohr T, Ohnesorge B, Bruder H, Stierstorfer K, Simon J, Suess C, et al. Image reconstruction and performance evaluation for ECG-gated spiral scanning with a 16-slice CT system. Med Phys. 2003 Oct;30(10):2650-62.

22. Flohr T, Ohnesorge B. Heart rate adaptive optimization of spatial and temporal resolution for electrocardiogramgated multislice spiral CT of the heart. J Comput Assist Tomogr. 2001 Nov-Dec;25(6):907-23. 
23. Manzke R, Grass M, Hawkes D. Artifact analysis and reconstruction improvement in helical cardiac cone beam CT. IEEE Trans Med Imaging. 2004 Sep;23(9):1150-64.

24. Grass M, Manzke R, Nielsen T, Koken P, Proksa R, Natanzon M, et al. Helical cardiac cone beam reconstruction using retrospective ECG gating. Phys Med Biol. 2003 Sep 21;48(18):3069-84.

25. Crawford CR, King KF, Ritchie CJ, Godwin JD. Respiratory compensation in projection imaging using a magnification and displacement model. Medical Imaging, IEEE Transactions on. 1996;15(3):327-32.

26. Desbat L, Roux S, Grangeat P. Compensation of some time dependent deformations in 2D tomography. In: Noo F, Zeng GL, Kudo H, editors. The 8th international meeting on fully three-dimensional image reconstruction in Radiology and Nuclear Medicine; 2005 July 6-9, 2005; Salt Lake City, UT; 2005. p. 129-32.

27. Ritchie CJ, Crawford CR, Godwin JD, King KF, Yongmin K. Correction of computed tomography motion artifacts using pixel-specific back-projection. Medical Imaging, IEEE Transactions on. 1996;15(3):333-42.

28. Roux S, bastien, Desbat L, Koenig A, Grangeat P. Exact reconstruction in 2D dynamic CT: compensation of timedependent affine deformations. Physics in Medicine and Biology. 2004(11):2169-82.

29. Parker DL. Optimal short scan convolution reconstruction for fanbeam CT. Med Phys. 1982 Mar-Apr;9(2):254-7.

30. Manzke R, Kohler T, Nielsen T, Hawkes D, Grass M. Automatic phase determination for retrospectively gated cardiac CT. Med Phys. 2004 Dec;31(12):3345-62.

31. Segars WP, Mahesh M, Beck TJ, Frey EC, Tsui BM. Computer-based simulation tools for high resolution x-ray CT research. Med Phys. 2006;33(Submitted).

32. Fung GSK, Segars WP, Taguchi K, Fishman E, Tsui BM. Development of a computer generated model for the coronary arterial tree based on multi-slice CT and morphometric data. SPIE Medical Imaging Conference 2006; 2006; San Diego, CA; 2006.

33. Segars WP, Taguchi K, Fung GSK, Fishman E, Tsui BM. Effect of heart rate on CT angiograph using the enhanced cardiac model of the 4D NCAT. SPIE Medical Imaging Conference 2006; 2006; San Diego, CA; 2006. 